\title{
On the Non-Optionality of Certain Modifiers
}

\author{
Sally McConnell-Ginet \\ Cornell University
}

\section{1 "Required" modifiers}

Middles, habituals and kind generics, many passives, and some adjectival passives seem to require either an overt adverbial or some other kind of verbal modifier. Although in many cases the "modification" may be contextually provided rather than overtly expressed, some kind of restriction on the class of eventualities designated by the main verb seems necessary (for well-formedness or felicity). Focusing on the so-called middle in English, this paper sketches the phenomenon and lays out some of the issues raised, suggesting lines for further inquiry. The major questions it raises are these:

(1) What defect(s) do middle modifiers remedy?

(2) How do they do it?

And it looks ahead to but does not explicitly address the larger question:

(3) What (if any) significant syntactic/semantic/pragmatic generalizations can be made about the defect(s) and remedies for different classes of expressions?

Although the discussion explicitly considers only English data, there is certainly evidence that modifier-neediness is not confined to English expressions (for cross-linguistic discussion of middles and the need for modifiers, see, e.g., Fagan 1992 and Fellbaum \& Zribi-Hertz 1989).

\subsection{Delimiting the data}

Here is a sampling of modifier-needy expressions in English. Although an asterisk is used in front of parentheses to indicate non-optionality of some expression like that in the parentheses (or in some cases of something provided contextually that functions like the parenthesized expressions), I do not thereby claim that missing modifiers result in ungrammaticality or even semantic illformedness. The requirement for modifiers may well be linked to pragmatic constraints (perhaps of a linguistically conventional kind, as suggested for any by Kadmon \& Landman 1993). Nor do I want to prejudge the question of whether the defect so indicated is exactly the same for all the kinds of expressions exemplified.

English middles need modifiers:

(1) Taurus handles *(well/like a racing car).

(C) 1994 by Sally McConnell-Ginet

Mandy Harvey and Lynn Santelmann (eds.), SALT IV 230-250, Ithaca, N.Y.: Comell University. 
(2) This toy assembles * (quickly/with only a screw driver).

(3) Babies comfort*(readily/with great difficulty).

(4) That piano plays *(superbly/in tune).

(5) Joan's dissertation reads * (well/like a mystery novel).

Stress or a negative or a modal or an interrogative can in some cases seem to provide what is needed:

(6) Now, this car HANDLES.

(7) She doesn't flatter.

(8) That piano should play.

(9) Does this dress wash?

As these examples suggest, contexts in which applicability of the middle without an overt modifier might distinguish among potential subjects (some dresses wash, others need to be dry-cleaned) are ones in which the middle might occur with no explicit indicator of restrictions. We might, e.g., answer (9) with (10a), which is roughly equivalent to (10b).

(10) a. Yes, this dress washes.

b. This dress is washable.

It is relatively rare, however, to find English middles without modifiers. Sentence (5) with no modifiers is just crashingly bad; it cannot be understood as equivalent to the well-formed (11).

(11) Joan's dissertation is readable.

Habituals seem to need modifiers:

(12) Mary walks a mile * (every morning/to work).

(13) Sandy looks in a mirror *(seldom/whenever bored)

Modifier-less habituals seem more readily rescued by context than middles. For example, (12) and (13) with no modifiers are fine if understood as answers to questions like (14) or (15).

(14) What does Mary do for exercise every day?

(15) How does Sandy always know who's walked in behind her?

Virtually any habitual seems rescuable contextually. In this respect, habituals contrast with middles like (5).

Passives sometimes seem to be modifier-needy:

(16) That house was built *(by my mother/to last/very solidly).

(17) This position is *(widely) held *(by many linguists) 
[Ex. from Grimshaw and Vikner 1993]

In the case of (16), one might speculate that the problem is that all houses are built (they don't just spring into existence); thus (16) is pragmatically deficient because it fails to distinguish "that house" from others. (We'll see that explanations of modifier-neediness in middles often also look to the requirement for classifying or distinguishing subjects.) Although some kind of pragmatic requirement to help draw distinctions may well be operative, it is not immediately clear how such an account can help with (17), given that (18) is fine (and can even be true).

(18) This position is held by no one.

Even more tellingly, (19) seems fine though, on the assumption that all houses are built, it is no more informative than the bizarre modifierless (16).

(19) That house was built by someone or other (or by some mechanical device).

Note, however, that many passives do not seem modifier-needy.

(20) Her home was destroyed.

(21) [Guess what!] Dewey was defeated/elected.1

Adjectival passives also sometimes need modifiers, even when the corresponding passive is not particularly modifier-needy:

(22) a. That is a *(well/un-) supported theory.

b. That theory seems to be supported.

The contrast between the adjectival passive (22a) and the verbal passive (22b) suggests that no purely pragmatic account of modifier-neediness will suffice.

Finally, we should note that not all obligatory adverbials are modifiers:

(23) Jan treated Chris *(badly/with great tact)

[cp. behaves]

(24) Pedro resides *(in Providence/with his mother)

[cp. put]

(25) The meeting lasted *(a long time/for an hour/until 7)

The manner, locative, and temporal expressions in (23)-(25) respectively are verbal arguments and not modifiers. They are semantically specific and selected by the verb. Letting $\mathrm{M}$ be a variable over "manners," we might, e.g., give behave a Davidsonian or neo-Davidsonian representation as in

(26) a. $\quad \lambda \mathrm{M} \lambda \mathrm{x} \lambda \mathrm{e}$ behave(M)(x)(e)

b. $\quad \lambda \mathrm{M} \lambda \mathrm{x} \lambda \mathrm{e}$ (behave(e) \& Manner(M)(e) \& Agent(x)(e)) 
Modifiers that rescue middles like those in (1)-(5) are not arguments of either the base transitive verb or the (derived) middle. Thus obligatory adverbials are not always diagnostic of modifier-neediness.

\subsection{Characterizing middles}

Let us turn now to look more closely at the syntactic and semantic structure of what we are calling middles in English.

\subsubsection{Transitivity alternations}

Middles are like passives and ergatives in being (at least superficially) intransitive counterparts of derivationally related transitives.

(1) a. Her hair combs beautifully.

b. The beautician combed her hair.

(2) a. Her hair was combed by the beautician.

b. The beautician combed her air.

(3) a. The lake froze.

b. She froze some fruit juice to put in the punch.

I assume without argument that middle formation is a matter of something like 1syntax (Hale \& Keyser 1993) or the "dynamic lexicon" (Fagan 1992). That is, there is some kind of regular word-formation process that shares many of its structural features with syntactic derivations but is nonetheless "lexical."

More specifically, middles, passives, and ergatives share property A:

A. The surface subject or external argument of the intransitive form (as in 1a-3a) is to be identified (in some way) with the internal argument (direct object) of the related transitive (as in $1 b-3 b$ ).

Many analysts have argued, however, that middles and ergatives differ from passives in having property $\mathrm{B}$ :

B. The surface intransitive does not express the external argument of the transitive.

Here is some evidence for that claim. Examples (2)-(4) show that agentive byphrases, purposive clauses, and adverbs assigning special properties to a "do-er" are allowed in passives but disallowed in both middles and ergatives.

(2) a. The book was read by many people.

b. The punch bowl was sold to raise money for medicine.

c. The car was handled skillfully.

(3) a. ${ }^{*}$ The book reads well by many people. 
b. *The punch bowl sold to raise money for medicine.

c. *The car handled skillfully.

(4) a. ${ }^{*}$ The ice melted by the sun.

b. *The ice melted to protect from falls.

c. ${ }^{*}$ The ice melted skillfully.

But Stroik (1991) and others have used examples like those in (5) to argue against B's holding for middles.

(5) a. The car handles well for Gina but not for Lou.

b. Articles about oneself never read well.

Zribi-Hertz (1992) argues that (5b) involves logophoricity; I recommend her discussion to those who think (5b) shows a syntactically present agent. She also claims that (5a) is to be explained without appeal to a syntactically present agent, though she seems to suggest that the for-phrase in (5a) is simply a beneficiary. As Stroik notes, (5a) does imply that Gina is somehow responsible for handling the car or "in control" of the car and not simply a beneficiary. In other words, Gina in some sense exhibits agency. But consider (6a), roughly equivalent to (6b).

(6) a. The baby sleeps for you but not for me.

b. The baby sleeps when you're in charge [or: on the scene] but not when I'm in charge.

Here too the for-object seems to be exerting "control" but there is no question of its being the agent of the event designated by the main verb. So sentences like (5a) do not provide evidence for the presence of an (implicit) agent of handle any more than sentences like (6b) provide evidence for an (implicit) agent of sleep.

If middles are like ergatives in not having an expressed agent, then just how do they differ from ergatives? First, ergatives are not at all modifier-needy:

(7) a. The ice melted.

b. The door opened.

And although (apparent) middles like those in (8) do not express agents, they do seem to entail active transitives like those in (9) and thus agents.

(8) a. Jane's exercise bike assembled very quickly.

b. Mother's punch bowl sold for $\$ 1000$.

(9) a. Someone/thing assembled Jane's exercise bike quickly.

b. Someone/thing sold Mother's punch bowl for $\$ 1000$.

In contrast the ergatives in (7) do not entail related causatives like those in (10) (although of course the converse entailments do hold).

(10) a. Someone/thing melted the ice. 
b. Someone/thing opened the door.

The sentences in (11) affirm those in (7) and deny those in (10).

(11) a. The ice melted by itself.

b. The door opened by itself.

In contrast, the sentences in (12) are quite bizarre.

(12) a. *Jane's exercise bike assembled very quickly by itself.

b. *Mother's punch bowl sold for $\$ 1000$ by itself.

Middles but not ergatives thus seem to have property C:

C. The surface intransitive incorporates reference to the same class of events as its active transitive counterpart; although no agent is expressed, agency of some kind is nonetheless semantically implied.

Many analyses treat the middle as somehow derived from its transitive counterpart, whereas many treat ergatives as basic intransitives from which causative transitives may be derived. This difference in derivation, of course, links to property $\mathrm{C}$.

\subsubsection{Two kinds of middles?}

Middles are (usually) generics, general statements rather than simple event descriptions. Notice, e.g., that the bare plural subjects in (1) cannot be interpreted existentially.

(1) a. Steinways play superbly.

b. Young babies startle readily.

These sentences ascribe properties to members of the kind rather than reporting events in which (perhaps only some) members participated. And this is true even when the middle does imply the occurrence of some particular event, as the past tense (2a), which entails (2b) IF the parenthesized material is omitted.

(2) a. Joan's dissertation read very well.

b. Someone read Joan's dissertation (very well).

c. Joan's dissertation was very good to read.

(2a) thus is equivalent to something like (2c); it presupposes, however, and does not assert the occurrence of an event like that reported in (2b) without the optional modifier.

But if we identify middles just on the basis of the transitivity alternations discussed above, then some middles do indeed seem to report events as the attested 
examples in (3) show:

(3) a. The punch bowl (finally) sold.

b. Flight 3870 (has) canceled.

The basic truth-conditions for the event-reporting middles in (3) seem indistinguishable from those for the passives in (4):

(4) a. The punch bowl was (finally) sold.

b. Flight 3870 has been canceled.

(1) and (2) contain the relatively common property middles; (3) contains what we'll provisionally call an eventive middle, much rarer in English.

Eventive middles seldom seem to need modifiers, as indicated by the optionality of the parentheses in (3). Yet eventives are like the property middles and unlike passives in having inaccessible agents; namely, property B discussed above. Here's another diagnostic for that inaccessibility. (5a) and (5b) are both appropriate after the passives in (4) yet only (5b) can follow the middles in (3).

(5) a. And just who sold/canceled it?

b. *By whom?

The syntactic inaccessibility of agents in eventive middles seems to provide counter-evidence to the proposal in Roberts (1986) that the need of middles for modifiers is due to the syntactic inaccessibility of their agents. If agent inaccessibility is what drives the need for modifiers, then eventives should differ from passives in needing modifiers. Apparently, however, the inaccessibility of agents does not result in eventives needing modifiers.

It seems that it is only property middles for which modifier need is an issue. The major reason for not calling eventive middles ergatives or (derived) unaccusatives is that they do seem to contrast with the usual instances of ergatives in semantically entailing the existence of an agent. But perhaps these intransitive eventives like sell and cancel in (3) are not lexically derived from their transitive homonyms in the same way as middles, being instead basic intransitive predicates. If so, then their implied "agents" may have a rather different status from those implied in property middles. If we take this line, then "agent inaccessibility" could be involved in explaining modifier-neediness. That is, property middles differ from so-called eventive middles in incorporating reference to an agent (though, crucially, not allowing that agent to be "accessible" to, e.g., by-phrase modification). In other words, a revised Property $\mathrm{C}$ might hold of property middles but not eventives. Notice that even events like ice melting or a door opening are "caused"; it's just that the cause need not be identifiable as some external agent. Likewise, the sale of a bowl or the canceling of a flight is "caused" but in such cases we find it hard to think of causes not readily identified as agents (no "by itself" seems possible, given the nature of the events), which 
may be why we are inclined (on this view, misleadingly) to think of these eventives as evincing Property $\mathrm{C}$ and thus being middles rather than ergatives.

Whether or not we adopt the view that eventives like those in (3) are to be classed as ergatives and not as middles, what is clear is that they lack both the generic modal character of prototypical "property" modals and their modifierneediness. And, as we will see below, eventive subjects are genuinely nonthematic, which seems not true of standard middle subjects.

\subsection{Middles and tough-movement}

Massam (1992) proposes that (non-eventive or "property") middles and tough-movement constructions are very similar, both syntactically and semantically; semantically, this proposal has considerable intuitive appeal. Suggestively, the tough-movement sentences in b. and the related impersonals in c. more or less paraphrase the middles in a.

(1) a. This bread cuts easily.

b. This bread is easy to cut.

c. It is easy to cut this bread.

(2) a. This bread toasts well.

b. This bread is good to toast.

c. It is good (satisfactory?) to toast this bread.

(c'. It produces good results to toast this bread)

(c". Toasting this bread produces good results.)

(3) a. Some silks wash.

b. Some silks are possible/feasible to wash.

(b'. Some silks can be washed.)

(b". Some silks are washable.)

c. It is possible/feasible to wash some silks.

Here are some further immediately obvious parallels:

A. Both kinds of sentences are stative.

B. Both have a surface subject identified with the null object position of a transitive verb.

C. Both "involve a secondary semantics which imposes a 'property' onto the derived [i.e., surface] subject." (Massam 1992, 126).

I'll discuss C. in more detail in my next section, but for now let us just note that this "secondary semantics" is absent from the impersonal tough-constructions in the c. sentences of (1)-(3) but present in both the middles in the a. sentences and their tough-moved counterparts in the $b$. sentences.

Massam's analysis suggests further parallels:

D. Both surface subjects are base-generated in what look like VP-external nonthematic positions [note that Massam's work does not incorporate the VP- 
shell hypothesis; what matters here is simply whether the surface subject is considered to be generated in some position distinct from that of the object position to which it is also in some way linked].

E. Both constructions involve a base-generated "empty (or resumptive) element in the thematic Case-marked (object) position." (126)

But she treats the empty object differently in the two cases:

F. In tough-constructions, the base-generated empty object moves to Spec of CP where it serves as an empty operator binding a (wh) trace in object position; in LF, the "non-thematic" subject is co-indexed with this A-bar chain by predication.

F'. In middles, the empty object is a null reflexive; i.e., a Case-marked anaphoric element requiring local co-indexing.

She argues that the object in middles is like that in recipe contexts:

(4) a. Take out the eggs ${ }_{\mathrm{i}}$ and [disc topic $]_{\mathrm{i}}$ break $[\mathrm{refl}]_{\mathrm{i}}$ into bowl.

b. *You take out the eggs ${ }_{i}$ and break [refl] into bowl.

The recipe-context null objects must be bound from a null subject position, filled in, Massam suggests by preceding discourse. Although the string in (4b) can be interpreted it has to be understood as linking the empty object to the you in subject position of the first clause, which preempts other discourse topics. The interpretation thereby produced of the second clause is, in fact a "middle," somewhat bizarre in having "you" broken into the bowl.

Most of Massam's arguments for the base-generated null object are syntactic, connected to such issues as the impossibility of middles with stranded prepositions, over ECM verbs, in double object constructions, etc. That is, middles don't really look syntactically as if there's been movement. Further support for the view that the middle subject itself is not base-generated in the direct object position comes from the impossibility of having idiom chunk object NPs as subjects of middles, in this way also paralleling tough-movement structures. Compare, e.g., (5a), (5b), (5c), and (5d).

(5) a. Honey keeps easily in glass jars.

b. *Tabs keep easily on foreign agents in Washington.

c. It is easy to keep tabs on foreign agents in Washington.

d. *Tabs are easy to keep on foreign agents in Washington.

There is thus strong support for the view that middles are control rather than raising structures.

It is also suggestive that many languages use overt reflexive morphology in middle constructions (e.g., French and German). Even English occasionally has overt reflexives in middle-like contexts. Massam gives examples like these: 
(6) a. This floor practically washes itself.

b. The analysis bases itself on the notion that ...

[Ex. from one of Massam's referee reports!]

The difference in the nature of the null objects in middles and toughconstructions should not obscure the critical parallel Massam draws in their identification.

G. The LF co-indexation of the "non-thematic" subject and the empty object is "licensed" by a tough-expression in INFL. In middles, the licenser is a modal (possibly phonologically null) or adverbial (moved into INFL at LF); in tough-movement, an adjective.

In a sense, what Massam is doing is locating the main "import" of middles not in the middle predicate itself but in some kind of modal and/or evaluative element. This element may sometimes be directly expressed by a modifier but in any case must be somehow linked to a modifier or an appropriate discourse-given element.

On this proposal, tough-constructions do involve movement but not of the lexical NP in subject position. Omitting irrelevant details and extrapolating from Massam's discussion, the proposed structure of (7a) seems to be something like (7b):

(7) a. This car is tough to handle.

b.

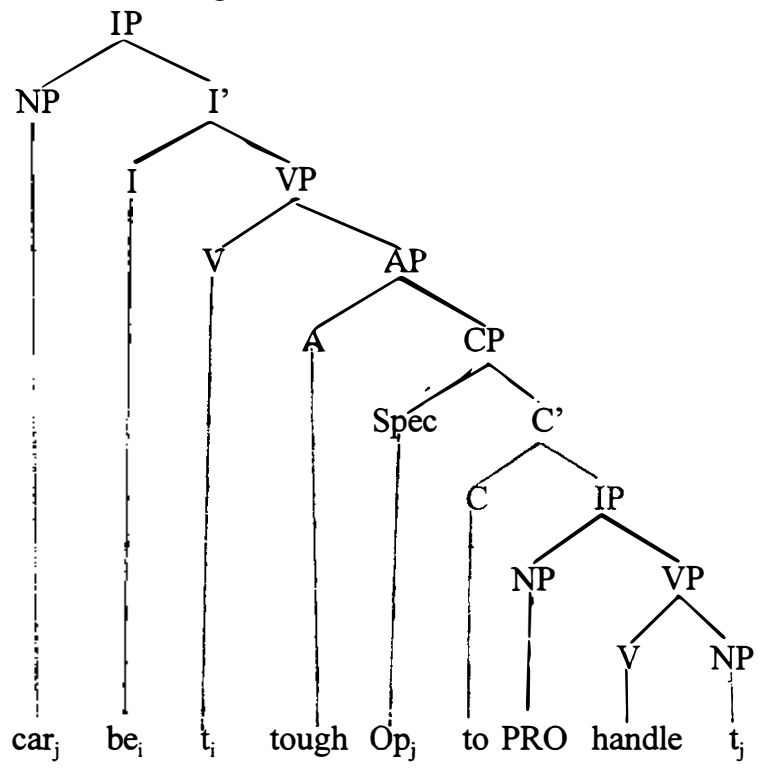

In contrast, middle (8a) has a structure something like (8b).

(8) a. This car handles easily. 


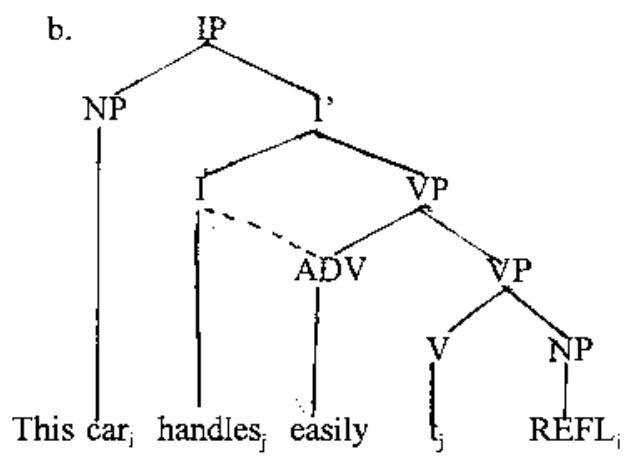

(The dotted line represents LF movement)

Massam's account has the virtue of treating modifiers that "rescue" middles as providing what is in some sense the main predicate of the middle construction, thus accounting immediately for their obligatory status. Questions remain. Can the "rescuing" be derived rather than stipulated? Why are particular elements able to rescue middles? Is the rescuer always a tough-element; i.e., an event-property or relation of an individual to an event? Under what conditions may modification be provided contextually? What is the relevance of the "secondary" semantics that seems to assign these "non-thematic subjects" some kind of significant property with respect to applicability of the tough-element (i.e., the implied feasibility, etc. of a process or satisfactoriness of results).

\subsection{The "secondary" semantics of middle subjects}

Middle (and tough-movement) subjects get interpreted not only as playing the thematic role associated with the internal argument of the input transitive verb (in the case of tough-movement, the embedded verb) but also as having a property by virtue of which they play some kind of causal or quasi-agent role. (See, e.g., van Oosten 1977, 1986; as noted in 2.1.2, this causally relevant property interpretation surfaces only in non-eventive middles.)

Note that (1a) is better than (1b), presumably because it makes explicit a causally relevant property.

(1) a. Small cars park easily.

b. ?Cars park easily.

c. It is easy to park cars.

And notice that the modifier that rescues the middles in (2) does so by suggesting that the subject is similar in its relevant properties to something else for which, presumably, we know characteristic effects produced as thematic participant in events of the kind designated by the base verb:

(2) a. Your dissertation read like a Harlequin romance. 
b. Since it's been tuned, the piano plays like a new instrument.

Of course, the rescuing modifier in (2) does two jobs: it not only attributes to the subject certain properties shared with the object of like but it also gives content to what Massam calls the tough-element, saying that events in which the subject is V-ed are (generally) similar in some important respect to events in which the like-object is V-ed, there being an assumption that we know what V-ing such objects is (generally) like.

Roughly, the "extra" semantic content of (1a) compared to (1c) seems to be something like this: some property of small cars is such that (the STATE of) their having that property is what CAUSES parking them to be generally easy. (1) says that small cars have a property that makes parking them generally easy; (1b) is odder than (1c) because (1b) requires us to impute some generic property to cars as a kind that makes (1c) true.

Letting $\mathrm{y}^{*}$ translate the null reflexive, something like (3) seems to represent what is predicated in (1) of small cars generally.

(3) $\lambda x \lambda e \cdot\left[\operatorname{easy}\left(\operatorname{parking}\left(y^{*}{ }_{i}\right)(e) \wedge x=\right.\right.$ Causer $\left.(e) \wedge x=y^{*}{ }_{i}\right]$

Causer is here being treated as a thematic role. If we let cause be a relation between event(ualitie)s, then we have something like (4a), where $P$ is a variable ranging over properties of individuals (cf. van Oosten's suggestion of "responsibility"); (4b) specifies the associated CAUSE relation of individuals to the events of which they are "Causer".

(4) a. $x=$ Causer(e) iff $\exists P[P(x) \&$ cause $(e)(P(x))]$

b. $\operatorname{CAUSE}(x)(e)$ iff $x=$ Causer(e) iff Causer( $x)(e)$

What we usually call Agents are Causers in this sense. To be a prototypical volitional Agent such as we find in activities and achievements requires further that the property in question be an action, something that the Causer DOES. To be a Causer for a generic middle--the thematic role we're hypothesizing for overt middle subjects--the property that CAUSEs the modal state (the capacity to be easily or quickly or satisfactorily Ved by an arbitrary agent) is presumably something like a disposition that helps generically characterize the kind of which the middle is predicated or, where predication applies just to some individual, a dispositional property in some sense characteristic of that individual (i.e., an individual-level or "generic" property of that individual).

Middles and tough-movement constructions are not the only place where the thematic role Causer seems relevant. Consider, e.g., subjects of transitives with Experiencer objects.

(5) a. The stillness frightened Robin.

b. Bureaucratic forms intimidate many people.

The subjects here are Causers; such verbs also, of course, readily take volitionally 
acting Causers (i.e., Agents) as their subjects. If such verbs take Causer subjects, we need say no more; as it happens, Causers for these kinds of eventualities may be Agents but need not be. Note, incidentally, that such verbs form middles:

(6) a. Robin frightens readily.

b. Many people intimidate easily.

Hale \& Keyser (1993) speculate that we may be able to replace thematic roles (e.g., "Affected Theme") by structural relations (e.g., case-marked internal subject). Taking this one step further, we might link the specificity of, e.g., Agent, to the presence at some level of representation of an aspectual operator DO (just a special case of CAUSE) in keeping with the spirit of Dowty (1979); Causer might just be the unmarked content of the subject of a CAUSE operator. I'll return this idea in the next section.

Leaving these large theoretical issues aside, however, the bottom line is that I am disputing Massam's characterization of middles (and tough-movement constructions) as having non-thematic subject positions. The middle subject position, I propose, receives a thematic role but not in the "main" expressed event, only in an imperfectly glimpsed and mostly implicit "sideshow."

\section{Middle formation}

Let's use as our example the middle in (1).

(1) Shakespeare translates easily.

Events of translating Shakespeare are provided with explicit agents in active transitive sentences like those in (2).

(2) Schiller translated Shakespeare.

Kratzer (1994) proposes that the VPs in such sentences will translate as in (3).

(3) גe.translate(Shakespeare)(e)

Roughly, the VPs will designate events of translating Shakespeare. In other words, besides the Davidsonian event argument the basic predicate translate needs only a single argument to saturate it; this argument is Hale \& Keyser's internal subject or "Affected Theme."

Hale \& Keyser's syntactic analysis treats the objects of transitive verbs like that in (2) as "internal subjects"; semantically, the verb plus object constitutes a complete or "saturated" predication that designates an event(uality) (or set thereof). The "external" subject is introduced to provide an argument to stand in the (abstract) cause relation to the event designated by the lower VP. To put it slightly differently, the operator [+caus] maps events into unary predicates holding of the Causers of such events. 
(4)

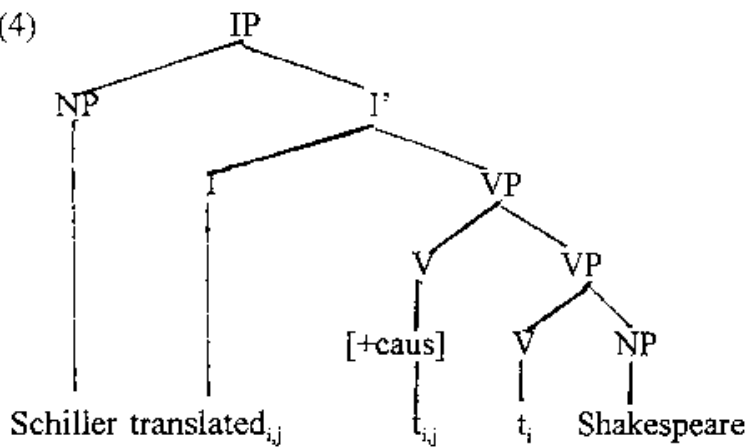

For Hale \& Keyser, it's plausible that the lower VP would receive an interpretation essentially like that in (3). Kratzer adds in the external argument position via a phonologically null functional head dubbed VOICE into which the verb moves; for Hale and Keyser, as I have said, this external subject arises as the argument for the phonologically null higher abstract causative verb that "incorporates" with the basic intransitive predicate translate to yield a transitive causative verb.

Something like (5) translates what Kratzer calls a VoiceP to which the subject NP will be adjoined.

(5) $\lambda x \lambda e \cdot[\operatorname{translate}($ Shakespeare)(e) \& Agent $(\mathrm{x})(\mathrm{e})]$

A strikingly similar interpretive approach works well for Hale \& Keyser's structure. For sentence (4), Hale and Keyser might want (6a) for the higher VP and (6b) for I' (if they were doing semantics).

(6) a. $\lambda$ e.cause(translate(Shakespeare))(e)

b. $\lambda x \lambda$ e.cause(translate(Shakespeare))(e) \& Causer(x)(e))

If we combine the subject NP with Kratzer's VoiceP and apply existential closure over the event variable we end up with (7); for Hale and Keyser, something like (8).

(7) $\exists e[$ translate(Shakespeare)(e) \& Agent(Schiller)(e)]

(8) $\exists e[$ cause(translate(Shakespeare))(e) \& Causer(Schiller)(e)]

Note that we are here using "cause" as the semantic correlate of Hale \& Keyser's abstract V [+caus], assuming that the VP it heads denotes an event of causing some other event--in this case, an event of causing Shakespeare to be translated. Such a cause itself takes an (external) argument, a Causer. We might, of course, want to require that the Causer be an Agent; i.e., that the Causer's DOing something be the event causing Shakespeare to be translated. And furthermore we certainly want some requirement about the immediacy of the cause. As was noted years ago (e.g., in Fodor 1970), a sentence like (2) is not synonymous with (9). 
The verb cause is vague in a way the causative verb translate is not with respect to the Causer's role; Schiller might be patron or master of the actual translator in (9) but not in (2) where Schiller must himself have done the job. And there are other ways this semantic interpretation of Hale \& Keyser's decomposition needs to be further developed. This should do for present purposes, however.

What then do we say about middles like (1)? Massam's analysis suggests that a lexical middle might have the structure represented in (10), where the active transitive is in some sense incorporated into the middle.

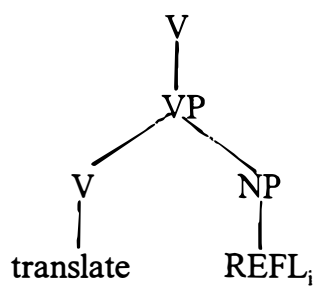

This derived intransitive $\mathrm{V}$ comes with its own internal argument, the phonologically null reflexive Massam posits. Let's assume that the derived V translates more or less as in (11).

(11) $\lambda$ e.translate $\left(y^{*}\right)(e)$

But of course this representation doesn't in itself disallow embedding under Kratzer's higher ActiveVoice head (or under Hale \& Keyser's abstract higher causative). Why couldn't we get sentence (1) equivalent to something like (12), where the generic operator (presumably introduced as it usually is in kind generics or habituals) has scope over an ordinary active voice verb?

\section{GN [translate(Shakespeare)(e) \& Agent(Shakespeare)(e)] [Easy(e)]}

Roughly, (12) says that events of Shakespeare's translating Shakespeare (the restrictor) are easy (the scope)--i.e., it's easy for Shakespeare to translate Shakespeare. (12), however, is not a possible interpretation for (1). Of course, (1) could be an active with an unspecified object; roughly, Shakespeare translates [stuff in general--whatever he tries to translate] easily. Shakespeare is the translator and we haven't really restricted what is translated. Or, as we've been assuming, it could be a middle that says Shakespeare is such that translating him [i.e., the stuff he wrote] is easy. But the intransitive verb with its null reflexive object that we've hypothesized as the form in middles can not be used in an active sentence with an external argument that is Agent of its designated event(s); i.e., (1) cannot be interpreted as equivalent to (12), with Shakespeare both translator and translated. 
What we want to end up with is a representation of sentence (1) along the lines of (13) (I'm using "s" to range over stative eventualities).

$$
\exists \mathrm{s}[\mathrm{s}=[\mathrm{GN}[\operatorname{translate}(\text { Shakespeare)(e)] [Easy(e)]] \& Causer(Shakespeare)(s) }
$$

If we follow the spirit of Hale \& Keyser's proposal, we might hypothesize that the (apparently intransitive) middle V already includes an abstract causative in its structure as well as the null reflexive object, with the structure looking like (14) rather than (10).

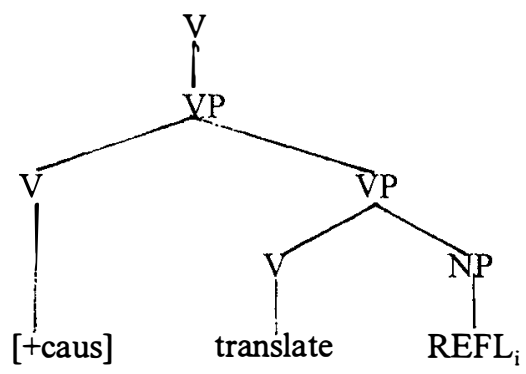

The impossibility of embedding such a V under an abstract causative could then be linked to the fact that ordinary transitive causatives don't themselves causativize (see Hale \& Keyser for discussion). (If we pursue Kratzer's framework, a similar story has to be told about why such a verb cannot embed under an ActiveVoice head.) In other words, we should provide (15) rather than (11) as a first approximation of a translation of (14).

(15) $\lambda$ e.cause $\left(\right.$ translate $\left.\left(y^{*}\right)(e)\right)$

We can for expository purposes, however, ignore this extra twist and assume the implicit causativity of our derived $\mathrm{V}$. What is crucial about having the causativity "inside" the single lexical item is that we cannot add that causativity in order to add an external argument of the (causative) translating. The lexical item already encodes in some sense a completely specified event (modulo the eventual specification of $y^{*}$ and the absence of any information about particular Agents or Causers of the "action").

Setting aside the eventives, which seem simply to be detransitivized and thus simple intransitives of the unaccusative or ergative variety, we find that middle constructions do attribute causality to their subjects. That causality, however, is not with respect to bringing about particular events of V-ing the subject; e.g., events of translating something (something that itself is to be identified with the subject). Rather the subject's causal role (e.g., Shakespeare's contribution) is with respect to such events' generically having some particular property--e.g., being easy or being good or being like events of V-ing some other kind of individual (recall, e.g., "this dissertation reads like a Harlequin romance" and similar cases). That is, the subject does not play a direct role in the event of 
V-ing something except as it serves to identify what is Ved; the thematic role of the subject is its causing events of V-ing it generically to have some property. This caused event-property is the analogue in middles of what Massam's toughelement designates.

Something like a MiddleOperator generically quantifies over a VP, which typically includes not only the derived intransitive $\mathrm{V}$ (with its internal null reflexive object) but also an adverbial that attributes some property to events of the sort designated by that V. Thus somewhere under IP we have a syntactic structure roughly like (16), where the derived V lexically includes a structure like that in (14).

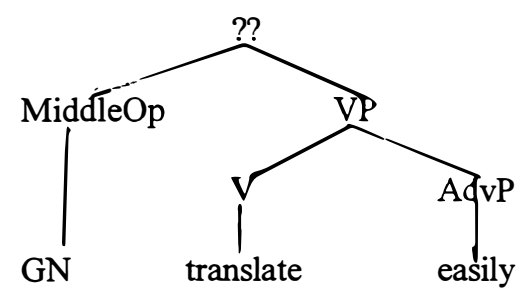

Structure (16) will be assigned an interpretation roughly as in (17).

$$
\lambda s\left[s=\left[G N\left[\operatorname{translate}\left(\mathrm{y}^{*}\right)(\mathrm{e})\right][\operatorname{Easy}(\mathrm{e})]\right]\right.
$$

Eventually we add in the wherewithal for a subject argument, perhaps at I'.

$$
\lambda \times \lambda s\left[s=\left[G N \text { [translate }\left(y^{*}\right)(e)\right][\operatorname{Easy}(e)]\right] \& \operatorname{Causer}(\mathrm{x})(\mathrm{s}) \& \mathrm{x}=\mathrm{y}^{*}
$$

Finally, of course, we add in the subject argument and apply existential closure and end up with something like what we proposed above in (13).

The representation in (13) indicates that what restricts the generic quantification in middles is the derived V: generalization is over events of translating whatever is to be added in as a subject. The adverbial predication then provides the nuclear scope for the generic operator--we are saying that, restricting attention to events of translating (it*), such events are easy.

Middles must have modification then in order to give a scope over which the generic quantifier can operate. The middle modifiers provide a predicate not unlike the tough-predicate. Although some of the content of the scope might be provided contextually, we cannot have a completely implicit nuclear scope. The scope, after all, is the major content of what is asserted. Restrictors for generic (and other) quantifiers are typically presupposed and thus can be provided contextually. That is, it's assumed that there's a non-empty class of events of translating Shakespeare; what's of interest is what such events are like. ${ }^{2}$

If modifiers in middles specify scope content for the generic quantifier, how can we ever have optional modifiers in middles? The rather neat picture inspired by the tough-movement parallel is complicated by cases where no explicit modifier is needed. (19) repeats some earlier examples. 
(19) a. This silk washes.
b. This dress buttons.
c. That car HANDLES.

Consider (19a) first. As we've noted earlier, it's one of a number of cases where the middle seems to come out more or less equivalent to the -able suffix. We don't presuppose events of silk-washing; rather we presuppose something like successful events of silk-cleaning and assert that such events generically can be events of silk-washing. As has often been noted in discussion of middles, cases like (19a) arise when there is a context of classifying things of the sort designated by the subject NP with respect to how suited they are to being Ved. (19b) is similar; the context must be something like consideration of successful events of closing up a dress and the assertion is that such events generically can be buttonings of the dress. The point is that the main verb is not needed as the restrictor because context has provided restriction via implicit contrast among different modes of doing something, the main $\mathrm{V}$ then being free to designate one such mode (serving then to give content to the scopal element).

The case of HANDLES seems somewhat different; handle (when it does not mean simply "manipulate with the hands") is similar to behave. It requires some kind of manner modification to saturate its argument structure. Like behave, however, handle can lack an explicit indication of the manner of handling (though for handle something like stress seems necessary) but will then be understood as including a positive evaluative implicitly ("handle well"). But since we need something like the "well" (implicit or explicit) for complete event specification we can't understand (19c) as saying that events of handling the car referred to are all good events. Instead (19c) seems to presuppose events of driving or something like that and asserts that such events are also events where the car is successfully "handled."

(19c) is similar to (20a), where the prepositional phrase occurring is not an event modifier but an argument that designates a participant in the event; note that the transitive (20b) requires the same class of locatives.

(20) a. This rack attaches *(to the wall).

b. Joan attached the rack *(to the wall).

Once we see that the locative is an argument and not our needed modifier, cases like (20a) look much like the examples in (19). The context provides some restrictor (events of successful/orthodox permanently locating the rack), and the sentence asserts that such events generically can be events of attaching it to the wall.

Thus in general modifiers are needed in middles to provide some explicit indication of the asserted scope--of the property being ascribed to events of the kind $\mathrm{V}$ designates. If a contextually provided restrictor frees up the $\mathrm{V}$ to function as part of the scope, this requirement may be relaxed and the $\mathrm{V}$ itself may provide content for the scope. This is likely to happen only when there is some question of whether a particular individual or kind of individual can be (successfully) V-ed, 
which is why modifierless middles generally indicate some kind of possibility. When the possibility of successful V-ing is not at issue, then successful V-ing (of an individual or kind to be identified with subject) is assumed and a property ascribed generically to such events.

\section{Conclusion}

Are there more than superficial resemblances between modifier neediness in the case of middles and in the others we've mentioned? Really to answer that question would require a much deeper and detailed account of middles and of the other cases where what look like modifiers appear to be non-optional.

Given that we've explained middle modifiers as needed to provide a nuclear scope for a generic operator by specifying an event-property, we'd expect to find some relation between modifier neediness in middles and in ordinary kind generics and habituals. Carlson (1989), however, argues that kind generics need modifiers mainly to help specify the restrictor, deriving scope content from either the predicate or the subject. Of course, if obligatoriness of modifiers is tied to their specifying scope and the possibility of contextual specification limited to identifying restrictors, the relative lesser modifier-neediness of kind generics as compared to middles would follow.

Grimshaw and Vikner (1993) proposed that passives need modifiers only when the internal structure of the events in question is "branching"--e.g., a process followed by some culminating state. But they do not really give an account of why modifiers are needed to "identify" these distinct event-parts nor how that "identification" is accomplished by the modifiers. Nor do they show why the passive should differ from the active in this respect. If it is agent-implicitness that matters, why and how does it matter? Perhaps here too modifier-neediness can be shown to depend on details of discourse presuppositions, syntactic structures, and the map they help induce to logical form.

Modifier-neediness in the verbal domain seems to force us to consider in some detail the semantic structure of events and event operators and the relation of syntactic structure to event-constructs. It also raises discourse-theoretic issues; here, we have mentioned presupposition and hinted at the relevance of other discourse-related phenomena.

\section{Endnotes}

* Comments from audiences at Cornell and at SALT 4 have improved this paper; as is customary, I absolve those commenters from responsibility for what I have done with their suggestions. I also thank Rosanne Pelletier for sharing with me an unpublished paper she wrote on the need of middles for modifiers at the beginning of her graduate career at Yale and Christine Fellbaum and Diane Massam for sending me their work on middles and related topics.

1. See Grimshaw \& Vikner 1993 for suggestions on how to characterize the 
contrast between passives that do need modifiers and those that don't; they also propose part of an answer to the question of why those modifiers might be needed. 2. Although her analysis of middles is somewhat different from what is proposed here, Condarovdi 1989 also sees middle modifiers as needed in order to give content to the nuclear scope of a generic. She does not, however, say anything about the conditions under which something else can specify scope content.

\section{References}

Condarovdi, C. (1989) "The middle: Where semantics and morphology meet", MIT Working Papers in Linguistics 11: Papers from the Student Conference in Linguistics, Dept. of Linguistics \& Philosophy, MIT.

Carlson, G.N. (1989) "On the semantic composition of English generic sentences" in Chierchia et al. (eds.), 167-192.

Chierchia, G., B.H. Partee, and R. Turner (eds.) (1989), Properties, Types and Meaning, V. II: Semantic Issues, Kluwer Academic Publishers, Dordrecht.

Dowty,D.R. (1979) Word meaning and Montague Grammar: The semantics of verbs and times in generative semantics and in Montague's PTQ, Reidel, Dordrecht.

Fagan, S.M.B. (1988) "The English Middle", Linguistic Inquiry 19, 181-203.

Fagan, S.M.B. (1992) The syntax and semantics of middle constructions: $A$ study with special reference to German, Cambridge University Press, Cambridge.

Fellbaum, C. (1985) "Adverbs in agentless actives and passives", Papers from the parasession on causatives and agentivity: Chicago Linguistic Society 21, 21-31.

Fellbaum, C. \& A. Zribi-Hertz (1989) "The middle construction in French and English: A comparative study of its syntax and semantics", Indiana University Linguistic Club.

Fodor, J.A. (1970) "Three reasons for not deriving 'kill' from 'cause to die', Linguistic Inquiry 1, 429-438.

Grimshaw, J. \& S. Vikner (1993) "Obligatory adjuncts and the structure of events" in Reuland \& Abraham (eds.), 143-155.

Hale, K. \& S.J. Keyser (1987), "A view from the middle", Lexicon Project Working Papers 10, Center for Cognitive Science, MIT.

Hale, K. \& S.J. Keyser (1993) "On argument structure and the lexical expression of syntactic relations", in Hale \& Keyser (eds.), 53-109.

Hale, J. \& S.J. Keyser (eds.) (1993) The View from Building 20, MIT Press, Cambridge.

Jaeggli, O. (1986) "Passive", Linguistic Inquiry 17, 587-622.

Kadmon, N. \& F. Landman (1993) "Any", Linguistics \& Philosophy 16, 353-422.

Keyser, S.J. \& T. Roeper (1984) "On the middle and ergative constructions in English", Linguistic Inquiry 15, 381-416.

Kratzer, A. (1994), "A compositional theory of the adjectival passive, Parts I and II", Handouts from March 1994 talk at Cornell.

Massam, D. (1992) "Null objects and non-thematic subjects", J. of Linguistics 28, 115-37. 
van Oosten, J. (1977) "Subjects and agenthood in English," Proceedings of the Chicago Linguistic Society 13, 459-471.

Reuland, E. \& W. Abraham (eds.) (1993) Knowledge and Language, v. II: Lexical and Conceptual Structure, Kluwer, The Netherlands.

Roberts, I. (1986), The Representation of Implicit and Dethematized Subjects, Foris, Dordrecht.

Stroik, T. (1992) "Middles and movement", Linguistic Inquiry 23, 127-137.

Wyner, A. (1994) Toward a restrictive theory of adverbial modification, Ph.D. dissertation, Cornell University.

Zribi-Hertz, A. (1993) "On Stroik's analysis of English middle constructions", Linguistic Inquiry 24, 583-589. 